\title{
Erratum
}

\section{A Flexible Synthesis of Indoles from ortho-Substituted Anilines: A Direct Synthesis of Isocryptolepine}

George A. Kraus, * Haitao Guo, Ganesh Kumar, Gerald Pollock III, Heather Carruthers, Divya Chaudhary, Jonathan Beasley Synthesis 2010, 1386.

The surname of author Heather Caruthers was misspelled in the author list. 